Historic, Archive Document

Do not assume content reflects current scientific knowledge, policies, or practices. 


\section{-}




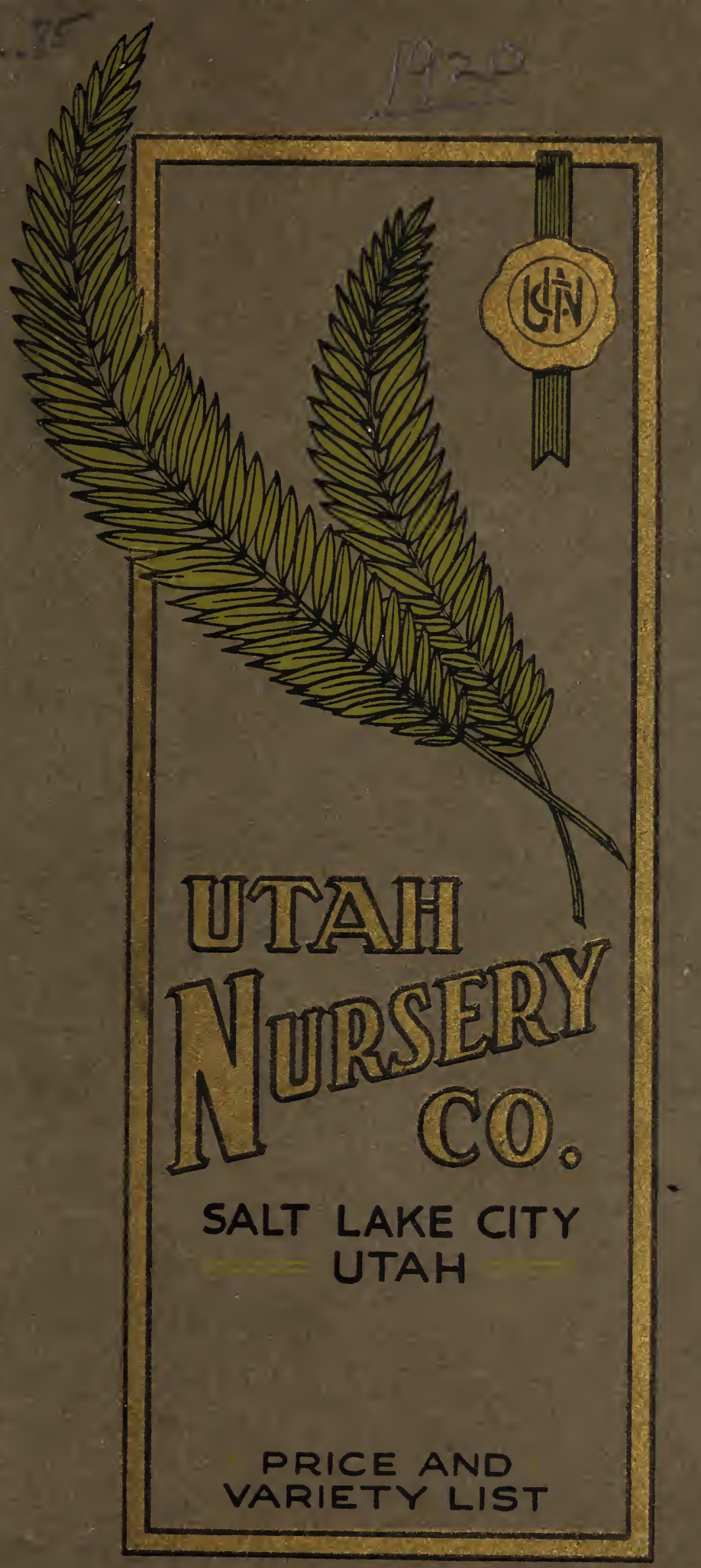


All prices and terms are subject to change without notice.

This is made necessary by unsettled business conditions that affect the cost of materials which we are obliged to buy for operating our business, and also by fluctuations in the prevailing wage rate.

THESE PRICES AND TERMS EFFECTIVE JULY 1ST, 1920.

Condensed catalogue price list. 


\section{FRUIT DEPARTMENT.}

\section{APPLES.}

One and Two Years. 4 Feet and Up.

$\begin{array}{cccc}\text { Singles } & \text { Dozen. } & 50 & 100 \\ \$ 1.25 & \$ 12.50 & \$ 40.00 & \$ 75.00\end{array}$

\section{SUMMER VARIETIES.}

Early Harvest-An old straw colored favorite. August.

Red June-(Early Strawberry). August.

Red Astrachan-Large, deep red, a fine cooker. August.

Yellow Transparent-Should be in every orchard. August.

\section{AUTUMN VARIETIES.}

Duchess-Yellow, streaked with red. September.

Gravensten-The great California Jelly apple. September.

Twenty-Ounce-Very large, streaked, cooking apple. September.

Maiden Blush-High quality, cream color, with pink cheek. September.

Mclntosh Red-A hardy red apple, grown in the north; finest quality. October.

Wealthy-In tre McIntosh class; fine. October.

\section{WINTER VARIETIES.}

Jonathan-The standard early winter apple. Red, rich. November, December.

Roman Beauty-Beautiful cream color, splashed with red; good keeper.

R. I. Greening-An old standard, known for its fine quality.

N. W. Greening-Very hardy, big cropper, good baker.

Winter Banana-Most beautiful in appearance, a leader on the market.

W. W. Pearmain-An old favorite, always in demand. Spitzenberg-One of the highest quality red apples. Gano-Large, bright red, big cropper, long keeper. Yellow Bellflower-Planted extensively in California. Black Twig-A Wine Sap type. Large, dull red; hardy.

Wine Sap-One of the leading commercial apples; dark red.

Stayman Wine Sap-Large, profitable on all markets, hardy.

Delicious-All that the name implies, possibly the best apple in the world today. Large, beautiful color, good bearer, hardy trees. Immune from blight. 


\section{CRAB APPLES.}

Transparent-Yellow with red cheek.

Whitney-Large, yellow, streaked with red.

Red Siberian-Very popular, yellow, splashed with red.

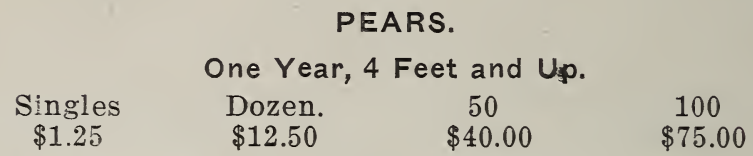

Bartlett-The leading commercial and domestic pear.

Flemish Beauty-Should be in every orchard; large russet or red; tree does well and crops heavy in most parts.

Seckel-Small russet, very rich.

Bure de Anjou-A fine early winter variety; large russet yellow.

P. Barry-A winter pear of fine quality; bears and keeps well.

Winter Nellis-One of the best winter pears, medium in size, strictly high class, russet, melting, rich.

\section{CHERRIES.}

One Year. 4 Feet and Up.

$\begin{array}{cccc}\text { Singles } & \text { Dozen. } & 50 & 100 \\ \$ 1.50 & \$ 15.00 & \$ 55.00 & \$ 100.00\end{array}$

Black Tartarian-Large, glossy, early cherry or known quality.

Windsor-Large liver colored flesh, fine quality. Sweet.

Napoleon-The leading sweet canning cherry; light red.

Bing-Purplish black, large, firm, sweet.

Lambert-Becoming the universal black sweet variety.

May Duke-Large dark red, mild, subacid, beautiful tree.

Royal Duke-In same class and character as May Duke. Subacid.

Early Richmond-Early light red, tart, cherry.

Montmorency-Large dark red, tart, popular with the market.

English Morello-Late, very dark when fully ripe. Subacid. 


\section{PLUMS AND PRUNES.}

One Year. 4 Feet and Up.

Singles

$\$ 1.25$

Dozen.

50

100

$\$ 12.50 \quad \$ 45.00$

$\$ 85.00$

Peach Plum-Fine, early, brownish red plum of quality; large.

Bradshaw-Large redish purple; a market favorite.

Green Gage-An old standard variety.

Yellow Egg-Very large, a plum universally known.

Lombard-Violet red, great cropper, very hardy, good.

Satsuma-The beet colored plum, large, very fine for jelly.

Italian Prune-Large dark purple; the commercial prune.

Frensh Prune-Medium in size, pink, very rich, fine for drying.

Hungarian Prune-Very large dark red, good on the market.

\section{PEACHES.}

One Year. 3 Feet and Up.

$\begin{array}{cccc}\text { Singles } & \text { Dozen. } & 50 & 100 \\ \$ 1.25 & \$ 12.50 & \$ 35.00 & \$ 60.00\end{array}$

Listed in about their order of ripening.

Red Bird Cling-New early red peach, fine for early market.

Alexander-Early dark red, has always been a leader.

Hales Early-A very fine red peach, following Alexander.

Triumph-The first yellow peach on the market.

Carmine-Beautiful cream colored peach with pink cheek.

Crawfords Early-One of the high class welloy varieties, freestone.

Fruitland Cling-New Orange Cling; promises to be a winn $\circ$. Ripens in September.

Tuscan Cling-California's leading canning peach; yellow. September.

Foster-Identical with Crawfords Early.

Earlv Elberta-Possesses all the qualities of the Elberta with additional fine color and highest quality; the best free stone yellow peach grown.

J. H. Hale-Large yellow freestone; new.

Elberta-The old standard shipper, hardy yellow free stone.

Late Crawford-A late orange free, of merit.

Utah Orange-An old favorite; fine color and flavor.

Chinese cling-White or cream with splash, very fine peach.

Sellers Cling-Fine color, large, firm and rich, yellow. October.

Health Cling-The leading late white cling; should be planted. October.

Philiips Cling-Very large, light yellow. 


\section{APRICOTS.}

\section{One Year. 4 Feet and Up.}

\section{Singles}

$\$ 1.50$

Dozen.
$\$ 15.00$

50

$\$ 55.00$

Moorpark-One of the finest Apricots; large yellow, with red cheek.

Colorado-In great demand in Colorado; large with fine color.

Blenheim-Fine, large, early variety.

Routier-A very large late Apricot, good quality.

Royal-The quality Apricot of California, a great canner.

Chinese-Supposed to be a Utah variety of high color and quality.

Tilton-Large yellow. Seems to be more exempt from late frosts than others.

\section{NECTARINES.}

One Year. 4 Feet and Up.

Fisher's Yellow Boston.

$\begin{array}{cccc}\text { Singles } & \text { Dozen. } & 50 & 100 \\ \$ 1.50 & \$ 15.00 & \$ 55.00 & \$ 100.00\end{array}$

\section{QUINCES.}

One Year. 3 to 4 Feet.

Champion Orange.

Singles Dozen.

$\$ 1.25 \quad \$ 12.50$

\section{NUTS.}

Almonds.

One Year. 3 to 4 Feet.

I X L Paper Shell. Ne Plus Ultra.

$\begin{array}{cccc}\text { Singles } & \text { Dozen. } & 50 & 100 \\ \$ 1.50 & \$ 15.00 & \$ 55.00 & \$ 100.00\end{array}$

Chestnuts. 
Walnuts.

Singles

Doz.

American Black, 4 ft. and up.... \$2.00

$\$ 18.00$

California Black, $4 \mathrm{ft}$. and up.... 1.25

12.50

English Walnuts, 2 to 3 ft...... 2.50

20.00

\section{Pecans.}

Pecan Seedlings, 2 to $3 \mathrm{ft} . \ldots \ldots 2.00$

\section{SMALL FRUITS.}

\section{Blackberries.}

\begin{tabular}{|c|c|c|c|c|}
\hline & Single & Doz. & 50 & 100 \\
\hline Lawton & $\ldots .25$ & $\$ 2.50$ & $\$ 7.50$ & $\$ 12.50$ \\
\hline Mersereau & .30 & 3.00 & 8.50 & 15.00 \\
\hline Vilson & $\ldots \ldots \ldots \ldots$ & 2.50 & 7.50 & 12.5 \\
\hline imalaya & Giant ... & 4.00 & 12.50 & 20. \\
\hline
\end{tabular}

\section{Raspberries.}

Cumberland

Single Doz. $\quad 50 \quad 100$

$\begin{array}{rrrrr}\text { Black Cap.....\$.30 } & \$ 3.00 & \$ 8.50 & \$ 15.00 \\ \text { regg Black Cap... } & .30 & 3.00 & 8.50 & 15.00 \\ \text { uthbert, Red........ } & 2.50 & 7.50 & 12.50 \\ \text { ouden, Red ........ } & 2.50 & 7.50 & 12.50 \\ \text { t. Regis, Red, } & & & . \\ \text { New Everbearing .... } & 3.50 & 11.00 & 20.00 \\ \text { oganberry ...... .40 } & 4.00 & 14.00 & 25.00\end{array}$

\section{Dewberries.}

Leucretia . ..... $30 \quad 3.00 \quad 8.00 \quad 15.00$

\section{Gooseberries.}

Oregon Champion

$\begin{array}{lllll}\text { (best of all) } \ldots & .35 & 3.50 & 11.00 & 20.00\end{array}$

\section{Grapes.}

One and Two Years.

$\begin{array}{cccc}\text { Singles } & \text { Dozen. } & 50 & 100 \\ \$ .60 & \$ 6.00 & \$ 22.50 & \$ 40.00\end{array}$


American Varieties.

Agawum

Concord

Moore's Early

Delaware

Niagara

Worden

European Varieties.

$\begin{array}{cccr}\text { Singles } & \text { Dozen } & 50 & 100 \\ \$ .50 & \$ 5.00 & \$ 16.00 & \$ 30.00\end{array}$

Flame Tokay

Thompson Seedless

Muscat or Alexander.

Currants.

Single Doz. $\quad 50 \quad 100$

Fay's Prolific, Red.\$ $.35 \quad \$ 3.50 \quad \$ 11.00 \quad \$ 20.00$

Lee's Prolific, Blk. .35

White Grape ..... . .35

Perfection, Red,

Ex. Good .... $\quad .50 \quad 5.00 \quad 15.00 \quad 27.50$

Strawberries.

$100 \quad 1000$

Marshall-Best for Utah.........\$2.50 \$15.00

Superb, Everbearing ........... $4.00 \quad 30.00$

Progression, Everbearing ........ $4.00 \quad 30.00$

Strawberry plants shipped in Spring only. Always shipped by mail or express. Not packed or shipped with other order.

MISCELLANEOUS.

\begin{tabular}{|c|c|c|c|}
\hline Each & Doz. & 50 & 100 \\
\hline Rhubarb .......\$.40 & $\$ 3.00$ & $\$ 13.00$ & $\$ 25.00$ \\
\hline Asparagus. . ...... & 1.50 & 2.50 & 4.00 \\
\hline
\end{tabular}


DECIDUOUS ORNAMENTAL.

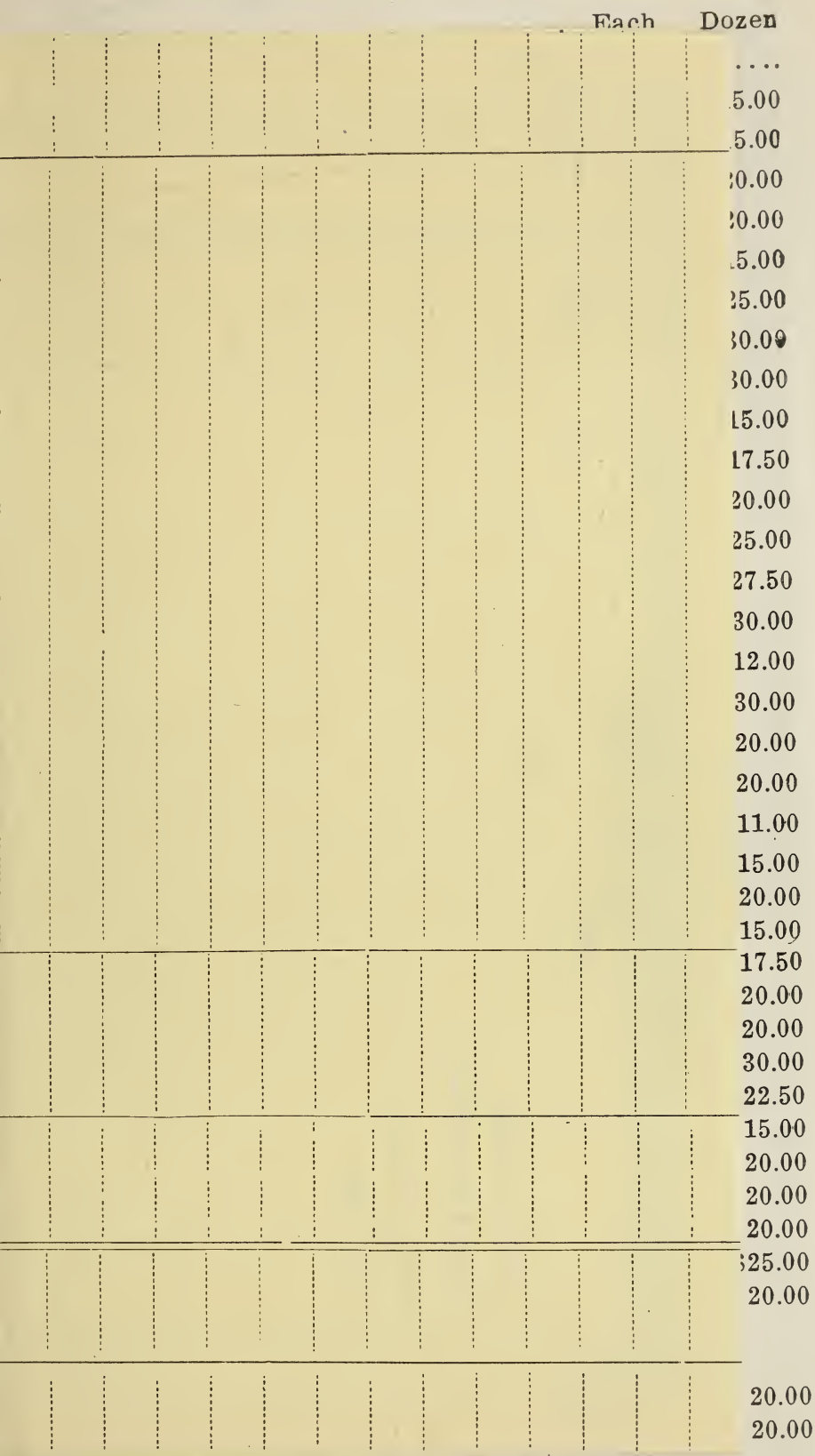

Per 50 Per 100 Per 1000

Locust, Black, 18 to 24 in......\$4.00 $\$ 7.50 \$ 50.00$

Locust, Black, 2 to $3 \mathrm{ft} \ldots \ldots \ldots 5.00 \quad 9.00 \quad 60.00$

Locust, Black, 3 to $4 \mathrm{ft} . \ldots \ldots . .8 .00 \quad 15.00 \quad 100.00$ 


\section{UTAH NURSERY CO.}

\section{Salt Lake City, Utah}

Date

PLEASE FILL T HE FOLLOWING ORLER:

NAME

\section{ADDRESS}

Ship to

Station

County of

State

Via

Mail Express or Freight

When to Ship

Order No.

AMOUNT ENCLOSED

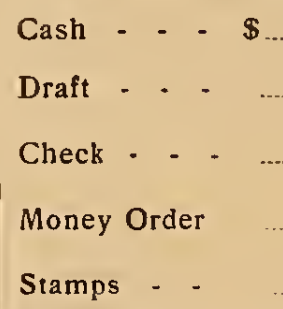

The Utah Nursery Company will exereise care to have all stock true to nane, nevertheless it is understood and agreed that should any stock prove untrue to name, the Utah Nursery Company shall be liable only for the sum paid for the stock which may prove untrue, and shali not be liable to any greater a monnt.

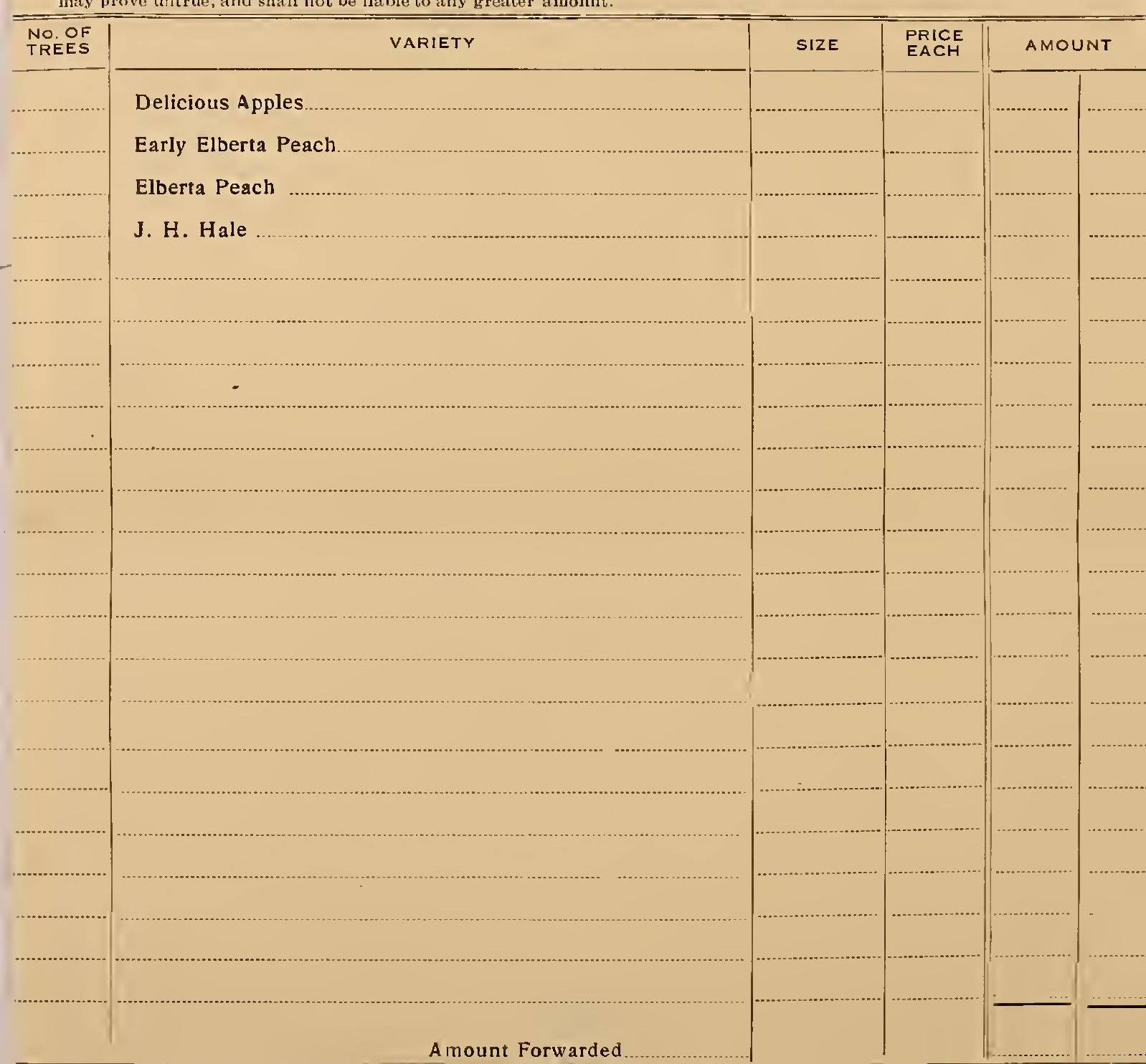


Amount Brought Forward 


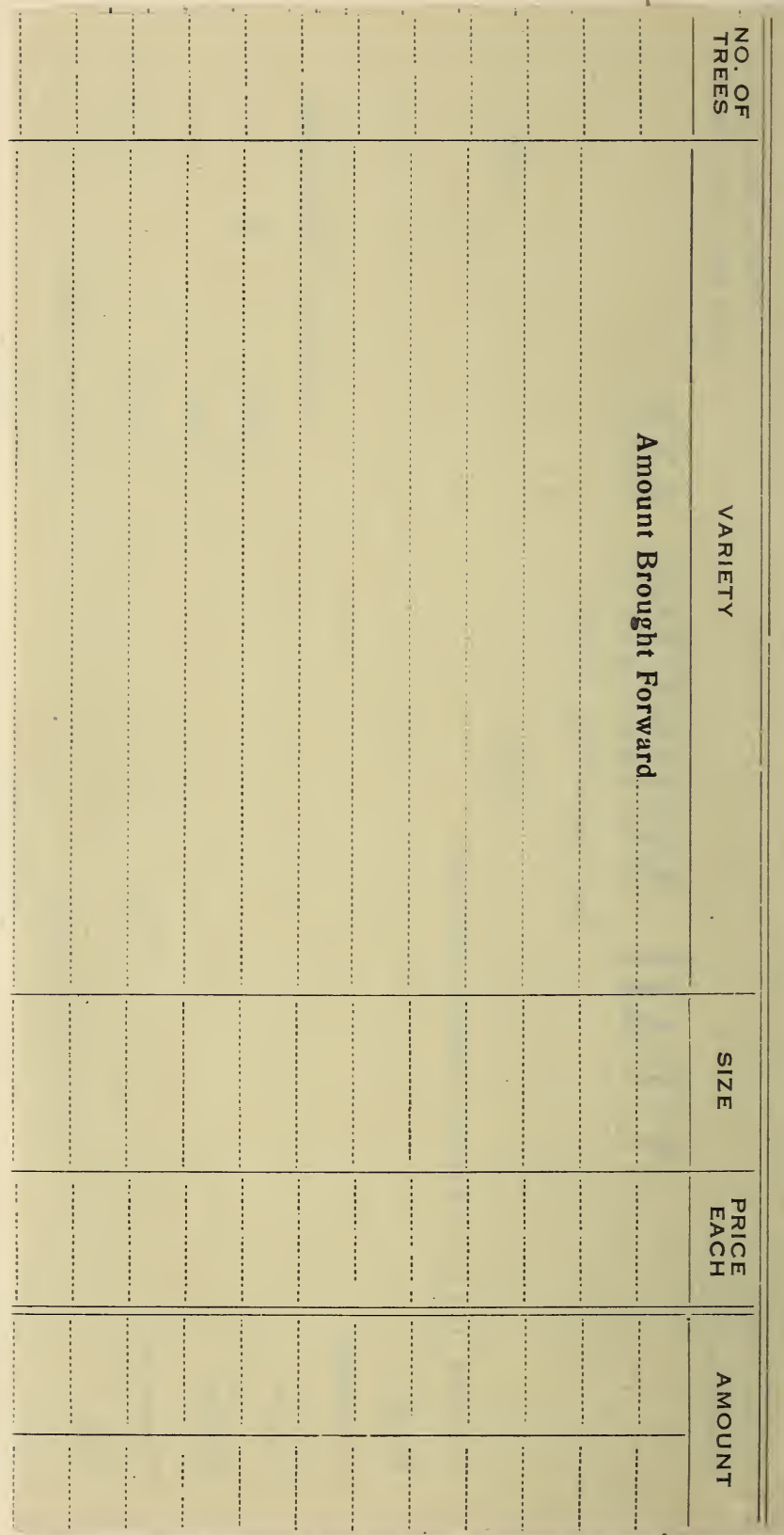


Each

Alder, European, $6-8$ ft........... $\$ 2.00$

Almond, dbl. flwr, pink, white, 3-4 ft... 1.50

Ash, European, 6 to $8 \mathrm{ft} \ldots \ldots \ldots \ldots 1.50$

Ash, Mountain, 6 to $8 \mathrm{ft} \ldots \ldots \ldots \ldots . .2 .00$

Ash, Mountain Oak Leaf, 4-6 ft...... 2.00

Balm of Gilead, 6 to $8 \mathrm{ft} . \ldots \ldots \ldots \ldots 1.50$

Beech, Standard European, 3-4 ft..... 2.50

Beech, Purple Leaf, 4 to $6 \mathrm{ft}$......... 3.00

Beech, River's Bronze Purple, 4-6 ft. . 3.00

Birch, Common White, 6 to $8 \mathrm{ft} \ldots \ldots 1.50$

Birch, Common White, 8 to $10 \mathrm{ft}$..... 1.75

Birch, Common White, 10 to $12 \mathrm{ft}$.... 2.00

Birch, Cut Leaf Weeping, 6 to $8 \mathrm{ft}$... 2.50

Birch, Cut Leaf Weep'g, 8 to $10 \mathrm{ft} . . .2 .75$

Birch, Cut Leaf, Weep'g, 10 to $12 \mathrm{ft}$.. 3.00

Box Elder, 6 to $8 \mathrm{ft} \ldots \ldots \ldots \ldots \ldots \ldots 1.25$

Catalpa, Bungei, 6 to $8 \mathrm{ft} \ldots \ldots \ldots \ldots . .3 .00$

Catalpa, Speciosa, 6 to $8 \mathrm{ft} \ldots \ldots \ldots 2.00$

Cherry, Double Flowering, 3-4 ft..... 2.00

Cherry, Dwarf Rocky Mountain...... 1.00

Crab, Bechtel's dbl. Flowering, 3-4 ft. . 1.50

Dogwood, variety, 3 to $4 \mathrm{ft} \ldots \ldots \ldots 2.00$

Elm, American or English, 6-8 ft..... 1.50

Elm, American or English, 8-10 ft... 1.75

Elm, American or English, 10-12 ft... 2.00

Elm, Cork Bark, 6 to $8 \mathrm{ft} \ldots \ldots \ldots \ldots 2.00$

EIm, Purple Leaf, 6 to $8 \mathrm{ft} \ldots \ldots \ldots 3.00$

Filbert, Purple Leaved, 3 to $4 \mathrm{ft}$. ..... 2.25

Fringe, White or Purple, 3 to $4 \mathrm{ft}$.... 1.50

Golden Chain or Laburnum, 6-8 ft.... 2.00

Honey Locust, 6 to $8 \mathrm{ft}$........... 2.00

Horse Chestnut, White, 4 to $6 \mathrm{ft}$..... 2.00

Horse Chestnut, Red flwrg, 4-6 ft . . . \$ \$2.50

Judas Tree, Red Bud, 3 to $4 \mathrm{ft}$....... 2.00

Laburnum, see Golden Chain.

Larch, European, 4 to $6 \mathrm{ft} \ldots \ldots \ldots \ldots 3.50$

Linden, Amer. or European, 6-8 ft.... 2.00

Linden, Silver Leaf, 6 to $8 \mathrm{ft} \ldots \ldots \ldots 2.00$
Dozen

$\$ \ldots .$.

15.00

15.00

20.00

20.00

15.00

25.00

30.00

30.00

15.00

17.50

20.00

25.00

27.50

30.00

12.00

30.00

20.00

20.00

11.00

- 15.00

20.00

15.00

17.50

20.00

20.00

30.00

22.50

15.00

20.00

20.00

20.00

$\$ 25.00$

20.00

20.00

20.00

Per 50 Per 100 Per 1000

Locust, Black, 18 to 24 in.....\$4.00 \$ 7.50 \$50.00

Locust, Black, 2 to $3 \mathrm{ft} \ldots \ldots \ldots 5.00 \quad 9.00 \quad 60.00$

Locust, Black, 3 to $4 \mathrm{ft} . \ldots \ldots . .88 .00 \quad 15.00 \quad 100.00$ 
DECIDUOUS ORNAMENTAL-(Cont'd.)

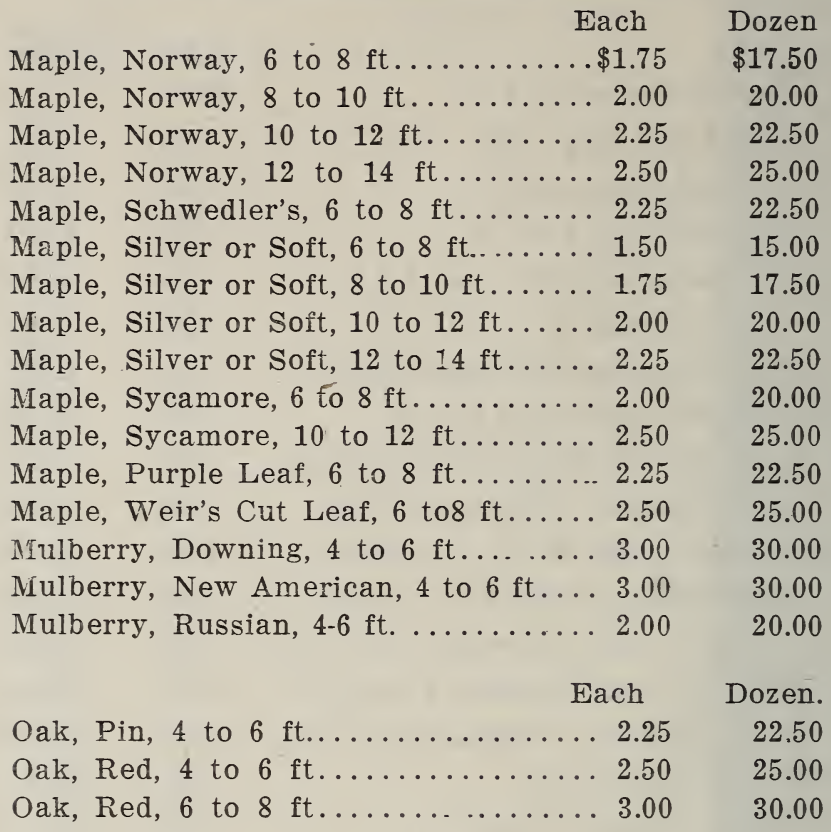

Poplar-

Singly P'er doz. Per 25 Per 50 Per 100

Carolina, 3-4 ft...\$... $\quad \$ \ldots \ldots . \quad \$ 8 \quad .00 \quad \$ 15.00 \quad \$ 25.00$

Carolina, $4-6$ ft... ... $\ldots . \ldots . \quad 12.00 \quad 20.00 \quad 35.00$

$\begin{array}{llllll}\text { Carolina, 6-8 ft... } & .75 & 8.00 & 15.00 & 27.50 & 50.00\end{array}$

Silver Lf., $6-8$ ft. $1.25 \quad 12.00 \quad 22.50 \quad 40.00 \quad 70.00$

Each Dozen

Plum, Pissardi, 4 to $6 \mathrm{ft} \ldots \ldots \ldots \ldots . \$ 1.50 \quad \$ 15.00$

Plum, Triloba, 3 to $4 \mathrm{ft} . \ldots \ldots \ldots \ldots \ldots 1.50 \quad 15.00$

Russian Olive (Elaegnus), 3-4 ft..... $1.50 \quad 15.00$

Salisburea (Maiden's Hair), 4-6 ft.... 2.50 25.00

Sweet Gum (Bilsted), 4 to $6 \mathrm{ft} \ldots \ldots .3 .00 \quad 30.00$

Sycamore, Oriental Plane, 6 to $8 \mathrm{ft} \ldots .25 \quad 22.50$

Thorn, Double Flowering, 4-6 ft..... 2.00 20.00

Tulip Tree, 4 to $6 \mathrm{ft} \ldots \ldots \ldots \ldots \ldots \ldots 2.25 \quad 22.50$

Willows, Golden, 3 to $4 \mathrm{ft} \ldots \ldots \ldots \ldots .1 .00 \quad 10.00$

WEEPING TREES.

Birch, Cut Leaf, Weep'g, 6 to $8 \mathrm{ft} \ldots \ldots \$ 2.50$

Elm, Camperdown ............ 4.00

Mountain Ash ............... 4.00

40.00

Willow, American ................ 1.75

17.50

Willow, Babylonica .............2.50 25.00

Willow Kilmarnock ............ $3.50 \quad 35.00$ 


\section{UPRIGHT DECIDUOUS SHRUBS.}

\section{Dozen rates are 10 times single price.}

Alithea (in variety) ...............\$1.25

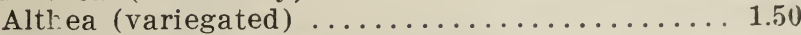

Azalea (Mollis), imported grafted...........4.00

Barberry, Purple Leaf, 2 to $3 \mathrm{ft} \ldots \ldots \ldots \ldots . . \ldots .75$

Barberry, Thunbergii .................. 75

Broom, flowering, White and Yellow ......... 1.00

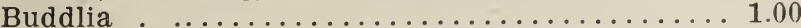

Blue Spiraea. (See Verbena Shrub.)

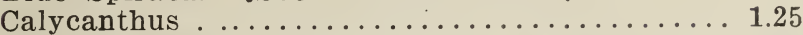

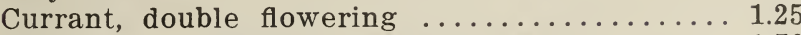

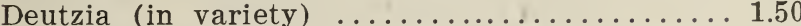

Elaeagnus (Silver Thorn) .............. 1.25

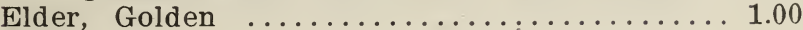

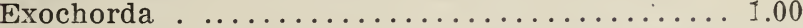

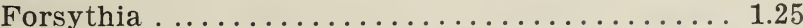

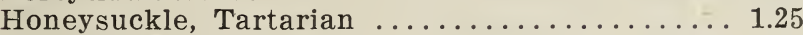

Hydrangea, Paniculata ............... 1.50

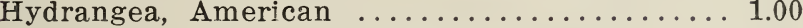

Hydrangea, all other varieties ........... 1.50

Hypericum (St. John Wort).............. 75

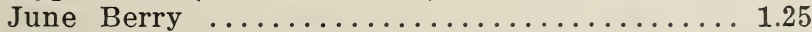

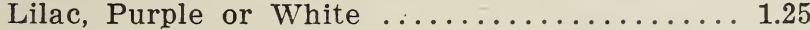

Lilac, all other varieties ............ 1.50

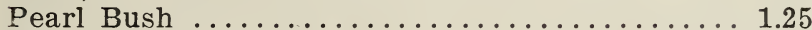

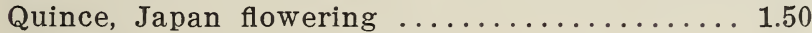

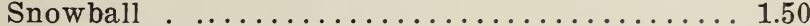

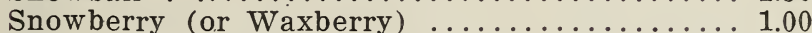

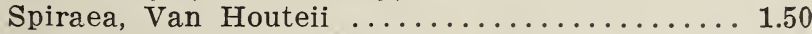

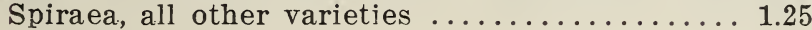

Svringa ...................... 1.50

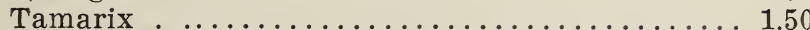

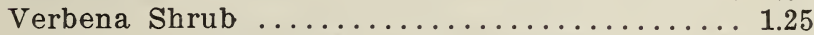

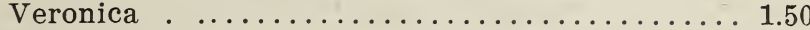

Weigela ........................ 1.50

\section{EVERGREEN SHRUBS.}

\section{Dozen rates are 10 times single price.}

Abelia, grandiflora ..................\$2.50

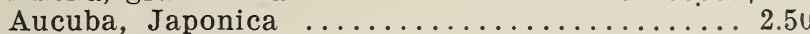

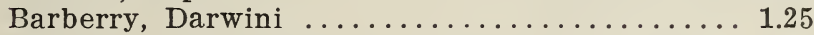

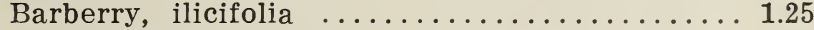

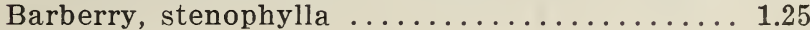

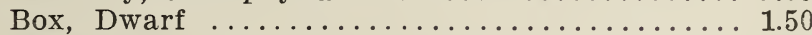

Box Tree .......................... 4.00

Camelia ....................... 4.00

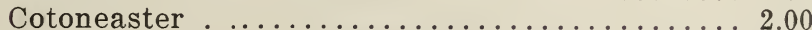

Daphne ........................... 4.00

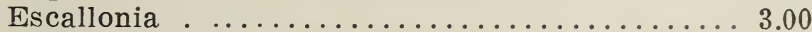

Euonymus ....................... 3.50

Holly, English, seedlings, 3 to $4 \mathrm{ft} \ldots \ldots \ldots \ldots \ldots 3.50$

Holly, grafted, berry bearing, 3 to $4 \mathrm{ft} \ldots \ldots \ldots 4.00$

Holly, grafted, variegated ............. 4.00

Japanese Evergreen Bittersweet............ 3.00

Laurel, Mt. (Kalmia), 18 to 24 inches.......... 3.50

Laurel, P'ortugal and English ............ 2.50

Laurustinus ..................... 2.50

Mahonia (Oregon Grape) ............... 2.00

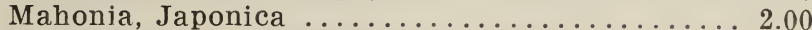

Pyracantha . ........................ 3.50 


\begin{tabular}{|c|c|}
\hline Per 100 & Per 1000 \\
\hline Berberis, 12 to 18 in.. & $\$ 150.00$ \\
\hline Dwarf Box, 6 to 8 in.. & \\
\hline Locust, Honey, 12 to 18 in..... & 75.00 \\
\hline Locust, Black, 12 to 18 in... & 50.00 \\
\hline Privet, Common, 12 to 18 in...... 12.00 & 100.00 \\
\hline Privet, California, 12 to 18 in..... 15.00 & .125 .00 \\
\hline
\end{tabular}

VINES AND CREEPERS.

Dozen rates are 10 times single price.

Ampelopsis Veitchii $\ldots \ldots \ldots \ldots \ldots \ldots \ldots \ldots \$ 1.00$

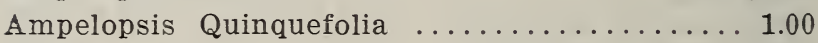

Aristolochia ............................. 1.00

Biguonia (Trumpet Vine) .............. 1.00

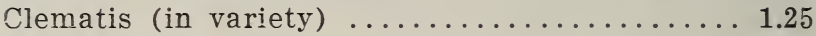

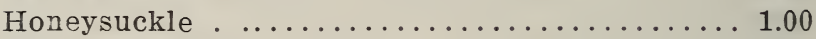

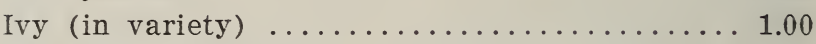

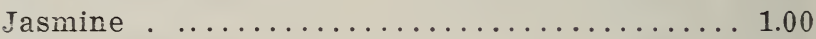

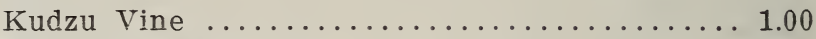

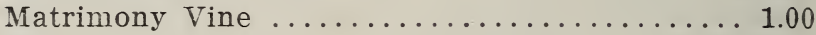

Virginia Creeper .................... 1.00

Wistaria .......................... 1.50

BULBS AND TUBERS.

Each. Doz.

Dahlia ...................\$\$ $\$ 3.00$

Gladiolus, assorted ............ $\quad 1.75$

Iris, assorted $\ldots \ldots \ldots \ldots \ldots \ldots \ldots \ldots . .35 \quad 3.00$

Jonquils . . . . . . . . . . . . . . .

Paeonias, assorted ............. $1.00 \quad 10.00$

All other varieties ............. $1.00 \quad 10.00$

(Sell no Tree Roses--only Bush Roses.)

NEW ROSES.

$\$ 1.50$ Each.

Los Angeles-This rose was introduced by Howard \& Smith in 1915. Already it is becoming a great favorite because of its own intrinsic worth. The color is absolutely new in roses.

Fire-Flame-Single flowers of flaming orange and copper, red. Strong grower and free bloomer.

Hoosier Beauty-Long buds and large, perfect flowers of ric' dazzling crimson on scarlet; fragrant. Vigorous growth and bloomer. 


\section{ROSES.}

- Each, \$1.00. Dozen, \$10.00.

American Beauty-Very popular as a greenhouse rose -not the best for garden.

Annie De Diesbach-Bright pink. Flowers are large.

Antoine Rivoire-Silvery flesh with salmon or carmine center. Excellent.

Baby Rambler-Dwarf crimson rambler.

Black Prince-Dark crimson, nearly black.

British Queen-Pure white. Growth vigorous.

Clara Watson-Silvery flesh-bears moderately in spring, but in wonderful profusion in fall.

Clio-Large flesh colored flowers; blooms continuously.

Edward Mawley-Brilliant fiery red. Free bloomer. Etoile de France-Velvet crimson. One of the best.

Frau Karl Druschki-Snow white-sometimes called White American Beauty.

Gen. Jacqueminat-Brilliant red; very popular.

Gen. McArthur-Fiery red. Continually growing more popular.

Gen. Superior Arnold Jonssen-Deep crimson.

Grauss an Teplitz-Rich scarlet. The ground color is intensely red, unsurpassed by any other. Unexcelled as a hedge rose.

Hadly-Excellent dark. Very fragrant.

Hermosa-Pink.

Hoosier Beauty-Long buds, large, perfect flowers of dazzling crimson.

Lady Hellingdon-Deep apricot yellow. One of the finest yellow roses.

La Detroit-Shell pink.

Milady-Crimson scarlet.

Mad. Caroline Testout-Bright pink. Also called Portland Rose.

Mad. Leon Pain-Silvery pink. Blooms freely.

Mrs. John Lang-Soft pink, large fine form. Good.

Ophella-Salmon flesh. Could hardly be praised too much.

Paul Neyson-Dark rose. The largest rose in cultivation for an outdoor rose; far excells American Beauty, for which it is sometimes mistaken. Strong and vigorous grower. 
Prince Camile de Rohan-Very dark crimson, large full flower. A good one to plant.

Ulrich Bruner-Extra strong grower; almost thornless. Cherry red; an old favorite.

Winnie Davis-F'lesh pink, outer petals silvery blush. An extremely heavy bloomer.

\title{
CLIMBING ROSES.
}

\author{
American Beauty \\ American Pillar \\ Crimson Rambler \\ Dorothy Perkins \\ Excelsa \\ Flower Fairfield \\ Lady Gay
}





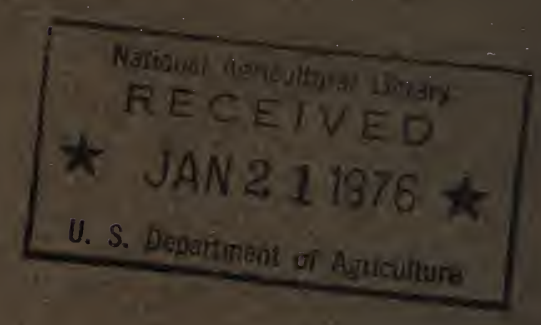

